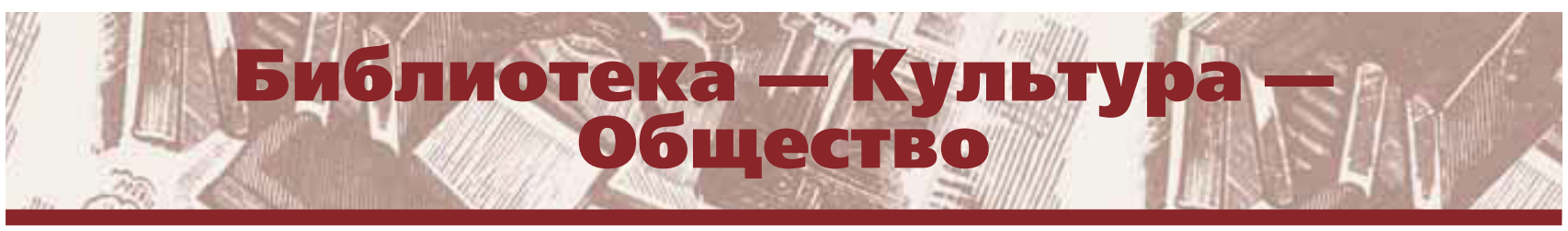

УДК 02

ББК 78.30

DOI 10.25281/0869-608X-2018-67-4-367-373

\title{
М.Я. ДВОРКИНА
}

\section{Разработка национального стандарта «Библиотечно- информационное обслуживание»: проблемы и решения}

Реферат. Статья посвящена актуальной теме - стандартизации, являющейся одним из факторов развития. Дано определение понятия «стандартизация», показано ее значение, порядок организации применительно к библиотечной деятельности. Рассказывается о разработке нового стандарта «Библиотечно-информационное обслуживание», начатой в 2017 г. в Центре по исследованию проблем развития библиотек в информационном обществе Российской государственной библиотеки. Выбор библиотечно-информационного обслуживания определяется тем, что этот важнейший вид библиотечно-информационной деятельности никогда не стандартизировался в межгосударственных и национальных стандартах. Цель разработки стандарта - упорядочить терминосистему библиотечно-информационного обслуживания, пересмотреть и уточнить определения терминов, отразить новые термины, связанные с информационно-коммуникационными технологиями. Необходимость разработки прежде всего терминологии библиотечно-информационного обслуживания связана с неоднозначностью трактовки большинства терминов. Представлена структура стандарта. Рассматриваются проблемы и дискуссии, возникшие при его создании, связанные с неоднозначным пониманием основного термина, понятий «вид», «форма» библиотечно-информационного обслуживания и услуг. Изложена последовательность разработки стандарта. Названы термины, отражающие использование современных информационно-коммуникационных технологий в библиотечно-информационном обслуживании. Указаны библиотеки и высшие учебные заведения культуры, которые наиболее активно участвовали в обсуждении стандарта и предложили свои замечания. Рассмотрен характер замечаний и предложений, содержащихся в отзывах на стандарт, названы некоторые принятые и отклоненные термины. Даны количественные характеристики принятых, частично принятых и отклоненных замечаний. Стандарт может использоваться для справок, поскольку он фиксирует современное представление о терминах библиотечно-информационного обслуживания, при планировании, отчетности и прогнозировании; он будет содействовать правильному обмену профессиональной лексикой между членами профессионального сообщества. Термины и определения, обозначающие базовые понятия библиотечноинформационного обслуживания, предназначены для применения работниками библиотечных учреждений, а также сотрудников служб научно-технической информации.

Маргарита Яковлевна
Дворкина,
Российская государственная
библиотека,
Центр по исследованию
проблем развития библиотек
в информационном
обществе,
главный научный
сотрудик
Воздвиженка ул., д. 3/5,
Москва, 119019, Россия
доктор педагогических наук,
профессор
E-mail: DvorkinaMY@rsl.ru


Ключевые слова: стандартизация, национальный стандарт, библиотечно-информационное обслуживание, библиотечное обслуживание, терминология.

Для цитирования: Дворкина М.Я. Разработка национального стандарта «Библиотечно-информационное обслуживание»: проблемы и решения // Библиотековедение. 2018. Т. 67, № 4. С. 367373. DOI: 10.25281/0869-608X-2018-67-4-367-373.

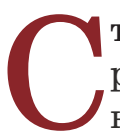
тандартизация - деятельность по разработке (ведению), утверждению, изменению (актуализации), отмене, опубликованию и применению документов по стандартизации и иная деятельность, направленная на достижение упорядоченности в отношении объектов стандартизации [1]. Стандартизация является одним из ключевых факторов, которые влияют на модернизацию, технологическое и социально-экономическое развитие страны [2].

Национальный орган по стандартизации в Российской Федерации - Федеральное агентство по техническому регулированию и метрологии (Росстандарт), создающее технические комитеты по стандартизации и координирующее их работу. Одним из технических комитетов (ТК 191) является комитет «Научно-техническая информация, библиотечное и издательское дело», образованный на базе Всероссийского института научной и технической информации (ВИНИТИ) PAH, занимающегося стандартизацией и в библиотечно-информационной отрасли. Проведение работ по стандартизации в области библиотечного дела осуществляет подкомитет 2 «Библиотечное дело», закрепленный за Российской государственной библиотекой (РГБ). Объект стандартизации в этой отрасли, на упорядочение которого стандартизация должна быть направлена, - библиотечно-информационная деятельность, одним из ее видов является библиотечно-информационное обслуживание [3, c. $67-70]$.

В 2017 г. в Центре по исследованию проблем развития библиотек в информационном обществе (ЦИПР) РГБ началась разработка терминологического стандарта «Библиотечно-информационное обслуживание». Была определена рабочая группа по созданию стандарта, в которую были включены, помимо представителей крупнейших библиотек (РГБ, Российской национальной библиотеки, Президентской библиотеки им. Б.Н. Ельцина, Государственной публичной научно-технической библиотеки (ГПНТБ) России, Библиотеки по естественным наукам РАН), преподаватели соответствующей дисциплины СанктПетербургского государственного института культуры.
Разработка стандарта велась в соответствии с законом «О стандартизации в Российской Федерации» [1] и основополагающими стандартами группы ГОСТ Р 1.0. Учитывались международные стандарты: ISO 5127:2017 Information and Documentation Foundation and Vocabulary (Документация и информация. Основные понятия и словарь), ISO 2789:2013 Information and Documen-tation International Library Statistics (Информация и документация. Международная библиотечная статистика), ISO 11620:2014 Information and Documentation - Library Performance Indicators (Информация и документация. Показатели функционирования биб-лиотек); а также национальные стандарты [4]: ГОСТ Р 7.0.20-2014 СИБИД. Библиотечная статистика. Показатели и единицы исчисления; ГОСТ Р 7.0.94-2015 СИБИД. Комплектование библиотеки документами. Термины и определения; ГОСТ Р 506462012 Услуги населению. Термины и определения; ГОСТ 7.0-99 СИБИД. Информационно-библиотечная деятельность, библиография. Термины и определения; ГОСТ 7.73-96 СИБИД. Поиск и распространение информации. Термины и определения.

Почему было решено разрабатывать стандарт по библиотечно-информационному обслуживанию и почему остановились именно на терминологическом стандарте? Выбор библиотечно-информационного обслуживания определяется тем, что этот важнейший вид библиотечно-информационной деятельности никогда не стандартизировался в межгосударственных и национальных стандартах. Необходимость разработки прежде всего терминологии библиотечноинформационного обслуживания связана с неоднозначностью трактовки большинства терминов.

Рассмотрим один пример. Из документа в документ на разных уровнях применительно к библиотекам используется понятие «библиотечное, библиографическое и информационное обслуживание». Но его содержание не осмысливается. Библиотечное обслуживание - это обслуживание, которое осуществляет библиотека, но некоторые понимают под этим термином выдачу документов, что позволяет считать, будто библиотека занимается только документо- 
выдачей. Библиографическое обслуживание предоставление библиографической информации, без указания на то, в каком учреждении оно осуществляется (в библиотеке, книжной палате, службе информации и др.). В библиотеке библиографическое обслуживание - вид библиотечного обслуживания. Информационное обслуживание - предоставление любой информации. Это обобщенное понятие, включающее обслуживание, реализуемое разными социальными институтами: библиотеками, архивами, музеями, книжными палатами, службами научной информации, средствами массовой информации, рекламными службами и любыми учреждениями, представляющими информацию о своей продукции. Следовательно, библиотечное обслуживание - один из видов информационного обслуживания. Возможно, поэтому все чаще употребляется термин библиотечно-информационное обслуживание. В стандарте библиотечное обслуживание и библиотечно-информационное обслуживание рассматриваются как синонимы.

Таким образом, в последовательность слов «библиотечное, библиографическое и информационное обслуживание» включены разноуровневые термины, которые не отражают смысл обслуживания в библиотеке. Стандарт должен помочь осознанию этого. Цель его разработки упорядочить терминосистему библиотечно-информационного обслуживания, пересмотреть и уточнить определения терминов, отразить новые термины, связанные с информационнокоммуникационными технологиями.

В рамках библиотечно-информационного (или библиотечного) обслуживания по разным признакам выделяются следующие виды обслуживания: документное, библиографическое, справочное, культурно-просветительское; индивидуальное, групповое, массовое, приоритетное; стационарное, внестационарное, дистанционное обслуживание и др.

На первом этапе разработки стандарта был составлен словник понятий, относящихся к библиотечно-информационному обслуживанию, содержащий свыше 200 терминов. Такой объем терминов обусловлен, в частности, тем, что существует большое число видов обслуживания, форм услуг. После рассылки всем разработчикам и получения предложений словник включал в себя более 500 терминов. Значительное место в нем заняли наименования различных форм мероприятий, а также синонимы некоторых представленных в первом варианте словника терминов. Столь значительный объем словника повлек за собой вопрос об отборе терминов для стандарта. Было решено отразить си- нонимы в примечаниях и сократить количество форм мероприятий до минимума, некоторые из форм были даны в качестве примера в определении понятия «библиотечное мероприятие». Это связано также с тем, что многие формы мероприятий сейчас только складываются (например, квест, литературный круиз, библиотечный караван), поэтому в стандарте остались только наиболее используемые формы услуг.

С учетом важнейших составляющих библиотечно-информационного обслуживания как вида деятельности [5] словник был структурирован по восьми разделам.

1. Общие понятия (содержит базовые термины: библиотечно-информационное обслуживание, библиотечно-информационная услуга и т. п.).

2. Пользователи.

3. Виды библиотечно-информационного обслуживания.

4. Библиотечно-информационные услуги.

5. Организационная структура библиотечно-информационного обслуживания.

6. Библиотечно-информационная среда.

7. Библиотечно-информационная технология.

8. Оценка качества и эффективности библиотечно-информационного обслуживания.

Позже от разделов 7 и 8 решено было отказаться, так как терминологически в них мало специфики, связанной с библиотечно-информационным обслуживанием, особенности имеются только на технологическом уровне. Однако в раздел 1 вошли некоторые базовые термины из разделов 7 и 8: «технология библиотечно-информационного обслуживания", «методы библиотечно-информационного обслуживания», «качество библиотечно-информационной услуги», «эффективность библиотечно-информационной услуги» .

Затем в разделе 7 решили дать статистические показатели. Но и от этого отказались, поскольку, с одной стороны, имеется ГОСТ Р 7.0.20-2014 «Библиотечная статистика: показатели и единицы исчисления» [4], в котором дан ряд статистических показателей библиотечного обслуживания, с другой стороны, по вопросам обслуживания очень много используемых в практике статистических показателей (что отражают и международные стандарты). Поэтому вместо включения этих показателей в терминологический стандарт целесообразнее их представить в новом варианте стандарта по библиотечной статистике.

Изменили название раздела 6 , поскольку понятие «библиотечно-информационная сре- 
да» относится не только к библиотечно-информационному обслуживанию, но и к деятельности библиотеки в целом. Термин «библиотечно-информационная среда» удален из данного стандарта с намерением включить его в более общий стандарт. Раздел 6 назван «Условия библиотечно-информационного обслуживания» .

В результате отбора в стандарте осталось 110 терминов, которым были даны определения, вместе с синонимами. Также представлены эквиваленты терминов на английском языке.

Определения некоторых терминов (например «вид библиотечно-информационного обслуживания») создавались заново, для формулирования дефиниции «форма библиотечно-информационной услуги» использовалась работа «Библиотечное обслуживание: теоретический аспект» [6, с. 243]. Для создания определений большинства терминов изучались материалы учебников [7], а также различных словарей, статей, стандартов, в том числе иностранных. Однако эти определения были уточнены и дополнены с учетом новых реалий.

В ходе обсуждения стандарта возникали дискуссии. Одна из них связана с термином «форма библиотечно-информационной услуги». Петербургские коллеги предлагали вместо понятия «форма» использовать понятие «вид». В качестве одного из важных аргументов правильности понятия «форма» применительно к конкретной библиотечно-информационной услуге московские разработчики использовали определение из «Краткой философской энциклопедии», согласно которой форма - это «внешнее очертание, наружный вид предмета, внешнее выражение какого-либо содержания, а также внутреннее строение, структура, определенный и определяющий порядок предмета или порядок протекания процесса в отличие от его “аморфного” содержания или содержимого» [8, с. 489]. То есть речь идет о чем-то устойчивом, сложившемся.

Именно такими сложившимися по своему строению являются формы услуг: выдача документов, выставка, викторина, вечер встречи и др. Поэтому по отношению к ним нужно использовать термин «форма». Проведенный автором анализ показал, что форма услуги показывает объект, который предоставляется пользователю, способы и/или условия, иногда - место его предоставления. Например, выставка предполагает демонстрацию (показ) документов (или их копий). Документы - объект; демонстрация, показ - способ.

Что касается термина «вид», то согласно «Краткой философской энциклопедии» он означает «понятие, которое образуется по- средством выделения общих признаков в индивидуальных понятиях и само имеет общие признаки с другими видовыми понятиями» [8, с. 67]. Так, видами услуг являются документные, справочные, культурно-просветительские и др. Вид объединяет формы услуг с одним объектом. Документные услуги (объект - документ) объединяют такие формы услуг, связанные с первичным документом, как выдача документа, доставка документов, бронирование документа и т. п. Несмотря на представленные аргументы, в разделе 4 стандарта пришлось отказаться от структуризации библиотечноинформационных услуг как форм.

Определенные разногласия возникли по поводу включения в стандарт термина «руководство чтением» [9], от которого все же большинство членов рабочей группы предложили отказаться как от устаревшего.

Были дискуссии относительно терминов «информационная грамотность» (используется в международных стандартах и литературе) и «информационная культура», который получил распространение в России благодаря работам Н.И. Гендиной [10]. В результате решено было основным термином сделать термин «информационная грамотность», а синонимом «информационная культура».

Проблемы возникли при определении понятия «виртуальный читальный зал». Это неожиданное словосочетание, но оно получило широкое распространение, поэтому от него решили не отказываться, хотя многие библиотекари виртуальный читальный зал связывают с реальным. Нежелание отказываться от этого термина определялось еще и тем, что разработчики ставили цель отразить новые термины, связанные с информационно-коммуникационными технологиями, но таких оказалось не очень много: «дистанционное обслуживание», «виртуальный читальный зал», «виртуальное посещение», «виртуальная справка», «виртуальное обслуживание», «выгрузка ресурса», «обслуживание через сайт библиотеки», «обслуживание через социальную сеть», «электронная услуга», «электронная доставка документов», «электронный абонемент» .

В апреле 2018 г. первую редакцию стандарта разослали на обсуждение в федеральные и региональные библиотеки, вузы культуры, а также разместили в Интернете. Было получено 24 отзыва на стандарт с замечаниями и множеством предложений (264) по его доработке. Наибольшую активность проявили: Кемеровский и Самарский государственные институты культуры, Государственная публичная истори- 
ческая библиотека России, Смоленская областная универсальная научная библиотека (ОУНБ) им. А.Т. Твардовского, Рязанская и Магаданская ОУНБ, Кемеровская ОНБ им. В.Д. Федорова, Фундаментальная библиотека Института научной информации по общественным наукам (ИНИОН) РАН, Научно-техническая библиотека Всероссийского научно-исследовательского проектно-конструкторского и технологического института электромашиностроения (ВНИТИ ЭМ), Научная библиотека Дальневосточного федерального университета, Централизованная библиотечная система ЦАО Москвы, Пермская государственная краевая универсальная библиотека им. А.М. Горького, Национальная библиотека Республики Карелия.

Значительная часть замечаний и предложений касалась определений терминов, предлагалось внести те или иные слова в дефиниции. Больше всего такого рода замечаний по определениям следующих терминов:

- «библиотечно-информационное обслуживание», «технология библиотечно-информационного обслуживания», «приоритетное обслуживание», «абонемент библиотеки», «кабинет для индивидуальной работы» (по 5 замечаний);

- «информационная грамотность», «поддержка чтения», «пользователь», «индивидуальное обслуживание», «внестационарное обслуживание», «обслуживание через социальную сеть», «выставочное обслуживание» (по 6 замечаний);

- «информационная потребность пользователя», «посещение», «виртуальное посещение», «обучение пользователей», «библиотечная консультация», «библиотечное мероприятие», «статистические показатели» (по 7 замечаний).

Предлагалось включить некоторые термины, которые отсутствовали в стандарте: «электронные услуги», «сетевое обслуживание», «обслуживание через сайт библиотеки», "редакция библиографического описания», «электронная справка», "регистратура», «электронная доставка документов» (этот термин был в стандарте, но не отдельно), «читательский интерес», «библиотечные практики». Из них в стандарт введены: «электронная услуга", "обслуживание через сайт библиотеки», «электронная доставка документов»; «виртуальная справка» (вместо «электронная справка»), «служба регистрации» (вместо «регистратура»). Термин «сетевое обслуживание» не включен в стандарт, поскольку уже имеется близкий по смыслу - «дистанционное обслуживание»; термин «редакция библиографического описания» отклонен, так как подобная услуга применяется редко; понятие «библио- течные практики» в библиотековедении почти не используется.

Полностью учтено $25 \%$ замечаний, частично - тоже $25 \%$, аргументированно отклонено $50 \%$ замечаний. В настоящее время подготовлена окончательная редакция стандарта. В перспективе возможно развитие, изменение и пополнение терминологии в новых терминологических стандартах.

Стандарт можно будет использовать для справок, поскольку он фиксирует современное представление о терминах библиотечно-информационного обслуживания, при планировании, отчетности и прогнозировании; он будет содействовать правильному обмену профессиональной лексикой между членами профессионального сообщества. Устанавливаемые им термины и определения, обозначающие базовые понятия библиотечно-информационного обслуживания, предназначены для применения работниками библиотечных учреждений и могут быть использованы сотрудниками служб научно-технической информации.

\section{Список источников}

1. Федеральный закон от 29 июня 2015 г. № 162ФЗ «О стандартизации в Российской Федерации» [Электронный ресурс] // Российская газета. 2015. 3 июля. № 6715(144). URL: https://rg.ru/2015/07/03/standart-dok.html (дата обращения: 05.07.2018).

2. Козлова Е.И., Антошкова О.А. Методические основы стандартизации в области библиотечно-информационного обеспечения науки. Стандартизация в Российской Федерации // Научная и техническая информация. Сер. 1. Организация и методика информационной работы. 2018. № 1. С. 26-32.

3. Дворкина М.Я.Библиотечно-информационная деятельность: теоретические основы и особенности развития в традиционной и электронной среде. Москва : ФАИР, 2009. 256 с.

4. Стандартизация в библиотечной деятельности : информ.-справ. пособие / Российская государственная библиотека. Технический комитет 191 «Научно-техническая информация, библиотечное и издательское дело» / [сост. и автор статьи Е.И. Козлова]. Москва : Пашков дом, 2018. $562 \mathrm{c}$.

5. Дворкина М.Я. Библиотечное обслуживание как система : учеб. пособие. Москва : Изд-во МГИК, 1992. $136 \mathrm{c.}$

6. Дворкина М.Я. Библиотечное обслуживание: теоретический аспект : (монография). Москва : Изд-во МГИК, 1993. 248 с. 
7. Библиотечно-информационное обслуживание : учебник / Ю.Ф. Андреева, О.Ф. Бойкова, В.А. Бородина и др. ; науч. ред. М.Я. Дворкина. Санкт-Петербург : Профессия, 2016. 240 с.

8. Краткая философская энциклопедия. Москва : Прогресс, 1994. 576 с.

9. Руководство чтением детей и юношества в библиотеке : [учеб. для библ. фак. ин-тов культуры, ун-тов и пед. ин-тов / Т.Д. Полозова,
Г.А. Иванова, Г.П. Туюкина и др.] ; под ред. Т.Д. Полозовой. Москва : Изд-во МГИК, 1992. $231 \mathrm{c.}$

10. Гендина Н.И., Колкова Н.И., Стародубова Г.А., Уленко Ю.В. Формирование информационной культуры личности: теоретическое обоснование и моделирование содержания учебной дисциплины. Москва : МЦБС, 2006. 512 c.

\title{
Development of the National Standard "Library and Information Services": Problems and Solutions
}

\author{
Margarita Y. Dvorkina, \\ Russian State Library, 3/5 Vozdvizhenka Str., Moscow, 119019, Russia \\ E-mail: DvorkinaMY@rsl.ru
}

\begin{abstract}
The article is devoted to the topical subject of standardization, which is one of the development drivers. The author presents definition of the concept Standardization, reveals its value and management procedure in relation to library activity. The article describes the development of new Standard "Library and Information Services", launched in 2017 at the Center for Study of the Development of Libraries in the Information Society of the Russian State Library. It is important to note that this type of library and information activities has never been standardized in international and national standards. The purpose of the Standard is to streamline the terminology system of library and information services, to revise and adjust term definitions and to reflect new terms related to information and communication technologies. The need to develop firstly the terminology of library and information services is associated with ambiguity of interpretation of most terms. The author presents the structure of the standard, considers the problems and discussions arisen at its creation and connected with ambiguous understanding of the basic term, of the concepts of "type" and "form" of library and information services. The author describes the sequence of development of the standard, names the terms reflecting the use of modern information and communication technologies in library and information services, indicates the libraries and higher educational institutions of culture, which most actively participated in the discussion of Standard and offered their comments. The article considers observations and proposals contained in the reviews of Standard, presents some accepted and rejected terms, as well as provides quantitative characteristics of the accepted, partially accepted and rejected remarks. The standard can be used for reference, as it captures modern understanding of the terms of library and information services, in planning, reporting and forecasting; it will facilitate the correct exchange of professional terminology among members of the professional community. Terms and definitions of the basic concepts of library and information services are intended for use by employees of library institutions, as well as by employees of scientific and technical information services.
\end{abstract}

Key words: Standardization, National Standard, Library and Information Services, Library Services, Terminology.

Citation: Dvorkina M.Y. Development of the National Standard "Library and Information Services": Problems and Solutions, Bibliotekovedenie [Library and Information Science (Russia)], 2018, vol. 67, no. 4, pp. 367-373. DOI: 10.25281/0869-608X-2018-67-4-367-373. 


\section{References}

1. The Federal Law of June 29, 2015, No. 162-FZ "On Standardization in the Russian Federation", Rossiiskaya gazeta [Russian Gazette], 2015, July 3, no. 6715(144). Available at: https:// rg.ru/2015/07/03/standart-dok.html (accessed 05.07.2018) (in Russ.).

2. Kozlova E.I., Antoshkova O.A. Methodical Bases of Standardization in the Field of Library and Information Support of Science. Standardization in the Russian Federation, Nauchnaya i tekhnicheskaya informatsiya. Ser. 1. Organizatsiya i metodika informatsionnoi raboty [Scientific and Technical Information. Series 1. Organization and Methods of Information Work], 2018, no. 1, pp. 26-32 (in Russ.).

3. Dvorkina M.Ya. Bibliotechno-informatsionnaya deyatel'nost': teoreticheskie osnovy i osobennosti razvitiya $v$ traditsionnoi i elektronnoi srede [Library and Information Activities: Theoretical Foundations and Features of Development in Traditional and Electronic Environment]. Moscow, FAIR Publ., 2009, 256 p.

4. Standartizatsiya $v$ bibliotechnoi deyatel'nosti: inform.-sprav. posobie [Standardization in Library Activities: information and reference manual]. Moscow, Pashkov Dom Publ., 2018, $562 \mathrm{p}$.
5. Dvorkina M.Ya. Bibliotechnoe obsluzhivanie kak sistema: ucheb. posobie [Library Service as a System: textbook]. Moscow, MGIK Publ., 1992, $136 \mathrm{p}$.

6. Dvorkina M.Ya. Bibliotechnoe obsluzhivanie: teoreticheskii aspekt: (monografiya) [Library Service: Theoretical Aspect: (monograph)]. Moscow, MGIK Publ., 1993, 248 p.

7. Dvorkina M.Ya. (ed.) Bibliotechno-informatsionnoe obsluzhivanie: uchebnik [Library and Information Service: textbook]. St. Petersburg, Professiya Publ., 2016, 240 p.

8. Kratkaya filosofskaya entsiklopediya [Brief Philosophical Encyclopedia]. Moscow, Progress Publ., 1994, $576 \mathrm{p}$.

9. Polozova T.D. (ed.) Rukovodstvo chteniem detei i yunoshestva $v$ biblioteke [Management of Children's and Youth Reading in the Library]. Moscow, MGIK Publ., 1992, $231 \mathrm{p}$.

10. Gendina N.I., Kolkova N.I., Starodubova G.A., Ulenko Yu.V. Formirovanie informatsionnoi kul'tury lichnosti: teoreticheskoe obosnovanie i modelirovanie soderzhaniya uchebnoi distsipliny [Creating Person's Information Culture: Theoretical Grounding and Content Modeling of an Academic Discipline]. Moscow, MTsBS Publ., 2006, $512 \mathrm{p}$.

\section{Aнонс}

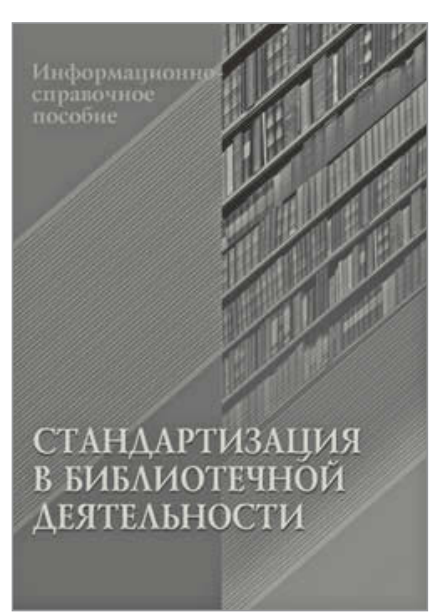

В день открытия 31-й Московской международной книжной выставки-ярмарки (05 сентября 2018 г., Москва, ВДНХ) на стенде РБА прошло подведение итогов конкурса «Лучшая библиотечная книга - 2018». Победителем в номинации «В помощь профессионалу» стало издание «Стандартизация в библиотечной деятельности».

Стандартизация в библиотечной деятельности : информационносправочное пособие / сост. Е.И. Козлова. Москва : Пашков дом, 2018. $563 \mathrm{c}$.

Издание содержит документы и справочную информацию о нормативном правовом регулировании библиотечной деятельности на основе системы национальной стандартизации. Включены все национальные стандарты в области библиотечного дела, утвержденные с 2013 по 2016 г., терминологические межгосударственные стандарты и стандарты по сохранности библиотечного фонда.

\section{Справки и заказ изданий:}

119019, Москва, ул. Воздвиженка, 3/5

Российская государственная библиотека, Отдел книжных изданий

+7 (499) 557-04-70, доб.25-72; Pashkov_Dom.Book@rsl.ru

http://store.rsl.ru/service/pashkov_dom 